\title{
EN DEFENSA DE LA IDENTIDAD DIFUMINADA: ESTRATEGIAS DE CREATIVIDAD Y PROMOCIÓN TURÍSTICA EN CIUDADES MEDIAS. EL CASO DE CASTILLA Y LEÓN ${ }^{1}$
}

\author{
Fernando Manero Miguel \\ Departamento de Geografía \\ Universidad de Valladolid
}

\section{RESUMEN}

Las ciudades constituyen escenarios de gran expresividad para entender el alcance de las transformaciones espaciales y culturales asociadas a la expansión del turismo contemporáneo. Debido a las estrategias puestas en práctica por el sector público y la iniciativa privada, la diversidad de experiencias revela los contrastes que distinguen a las ciudades entre sí en función de la desigual magnitud de sus recursos y de su capacidad para valorizarlos de acuerdo con los desafíos impuestos por la demanda. En este panorama diferenciado, cabe resaltar el peso progresivamente alcanzado por las ciudades que, situadas en el nivel intermedio de la jerarquía urbana, acometen la realización de proyectos basados en la recuperación de sus bienes patrimoniales históricos y en la creación de ofertas innovadoras, a fin de reforzar su imagen identitaria con la proyección de sus atractivos a gran escala. Las experiencias llevadas a cabo en las ciudades capitales de provincia turísticamente menos dinámicas de la Comunidad Autónoma de Castilla y León ofrecen un buen ejemplo de esta tendencia.

Palabras claves: Patrimonio cultural, Turismo Urbano, Ciudades Medias, Desarrollo Local, Políticas Urbanas.

\begin{abstract}
Cities are very expressive scenarios to understand the spatial and cultural transformations associated with the expansion of contemporary tourism. Due to the strategies implemented by the public sector and private initiative, the diversity of experiences highlights the contrast that distinguish cities from each other terms of the unequal size of their resources and their ability to value them according to the challenges of demand. In this differentiated context is interesting to note the progress made by cities

1 La realización de este trabajo se inscribe en la línea de investigación amparada en el Proyecto «Nuevas metodologías y directrices aplicadas a la ordenación y gestión del territorio: estudio comparado de las políticas territoriales de Castilla y León en el contexto español e internacional» (VA025A08), financiado por la Junta de
\end{abstract} Castilla y León. 
situated in the intermediate range of the urban hierarchy in the implementation of projects based on the recovery of historic heritage assets and the creation of innovative offerings in order strengthen its identity with the projection image of the attractions on a large scale. Experiments realised in the capital cities of less dynamic tourist provinces of the region of Castilla and Leon are a good example of this trend.

Key Words: Cultural heritage, Urban Tourism, Middles Cities, Local Development, Urban Policy.

\section{Introducción}

Si la valoración de las estrategias de desarrollo socio-económico promovidas por las ciudades reflejan lógicas coincidentes y están justificadas por factores que generalmente responden a los mismos fines, la experiencia revela que las posibilidades para alcanzar tales objetivos no se sitúan en todos los casos en condiciones de igualdad. Las economías de escala territoriales más vigorosas resultan, en principio, determinantes a la hora de favorecer la aplicación de estrategias que, al tiempo que optimizan los recursos de toda índole disponibles con ese fin, aseguran sus respectivas capacidades y permiten afrontar las situaciones críticas que inevitablemente inciden en las causas a las que se deben las variaciones en los procesos de crecimiento. Consiguen así un nivel de confianza frente a las incertidumbres del futuro mucho mayor del que, en cambio, caracteriza las perspectivas a medio y largo plazo de los elementos urbanos que adolecen de carencias estructurales o no poseen un soporte funcional lo suficientemente sólido para consolidar sus fortalezas en el marco de relaciones, fuertemente competitivo, en el que necesariamente han de desenvolverse.

Ahora bien, esta diferenciación, que resulta patente cuando se refiere a las situaciones de disparidad estructural - por lo que respecta a su entidad demográfica o al fortalecimiento de sus respectivas economías de escala, magnitudes inherentes a las estrategias urbanas contemporáneas (Law, 2002, 4) - debe ser revisada cuando la atención se centra en el análisis de los comportamientos espaciales asociados a la actividad turística, donde esta dicotomía puede verse sensiblemente matizada. Pues no es irrelevante, en efecto, el papel desempeñado por las ciudades de pequeño y mediano tamaño en el panorama de las tendencias que definen actualmente los procesos de transformación inducidos por el turismo, en los que han logrado, no sin esfuerzos y dificultades aunque con un alto grado de compromiso, alcanzar posiciones muy reconocidas. La experiencia revela con claridad hasta qué punto muchas de ellas han acreditado su prestigio hasta afianzarse como referencias fundamentales en el elenco de los lugares de atracción que se abren al interés y a la curiosidad del visitante potencial, del mismo modo que en ocasiones han llegado incluso a marcar las pautas en las que deben encuadrarse las directrices que garantizan los resultados positivos consecuentes a una estrategia bien concebida y, a la postre, acertada. Una estrategia que no es ajena al objetivo que la anima en el sentido de procurar, mediante el turismo, al amparo de la imagen construida con tal fin (García Martínez, 2011, 438) y sobre la base de la importancia asignada a esta actividad en el contexto de las políticas urbanas (Calle, 2008, 509), impulsar el desarrollo de las ciudades y su propio beneficio sin que ello implique la merma de identidad, riesgo sobre el que acertadamente advertía el Consejo de Europa en la Declaración de Dubrovnik (2006)².

2 Las reflexiones planteadas en este artículo se fundamentan en la necesidad de dar respuesta, a través del análisis empírico, al desafío recogido en la Declaración de Dubrovnik, surgida a raíz del Symposium Internacional -Cultural Tourism: Economic Benefit or Loss of Identity? - en el que se suscitó la disyuntiva de que son susceptibles los impactos del Turismo Cultural en los espacios urbanos. Evidentemente, se trata de una cuestión de gran importancia que invita al estudio comparado sobre la base de las numerosas experiencias que pueden decantar las conclusiones en una u otra dirección. 
Contemplado desde esta perspectiva, y teniendo en cuenta la importancia que se le concede entre las grandes líneas de acción de la Unión Europea (Proyecto PICTURE, 2003-2006), el fenómeno presenta numerosos matices, que ilustran sobre la variedad de situaciones de que es susceptible la interpretación de los vínculos surgidos entre turismo, desarrollo económico y espacio urbano, cuyo significado geográfico remite no sólo a la calidad y relevancia de los bienes valorizables como recursos efectivos sino también a los instrumentos de intervención aplicados a partir de la voluntad y sensibilidad manifestadas por los distintos actores para asumir y aprovechar las oportunidades que se presentan, y que a su vez derivan de la confluencia de las perspectivas que cada uno de ellos posea de la ciudad y de sus posibilidades como espacio de atracción turística. En definitiva, todo ello no es sino la expresión económico-espacial del engarce que cabe establecer entre los elementos susceptibles de ser utilizados y la solidez de la estructura de soporte sobre la que se sustenta la estrategia de rentabilización aplicada a ellos con fines turísticos (Virassamy, 2002,22 ), por cuanto en las ciudades situadas en la escala intermedia del sistema urbano se asume también con firme voluntad política la necesidad de aprovechar las posibilidades consustanciales al turismo como uno de los factores esenciales del desarrollo económico (Selby, 2004, 10). De ahí que esta estructura evolucione y se transforme a partir de las interrelaciones producidas entre las políticas públicas orientadas específicamente al impulso del sector, el entramado empresarial, que se configura a su vez sobre la base de la cooperación público-privada, y el complejo formado por los proyectos en los que cristaliza la capacidad de iniciativa desplegada. Asimismo las sinergias que entre estos componentes se generan no pueden entenderse al margen de los flujos financieros y de las directrices que encauzan los planes de promoción y marketing. En suma, un entramado coherente (Fig. 1) que convierte al turismo en una actividad de importancia sustancial para interpretar la ordenación integral del espacio construido (Reysset, 2008).

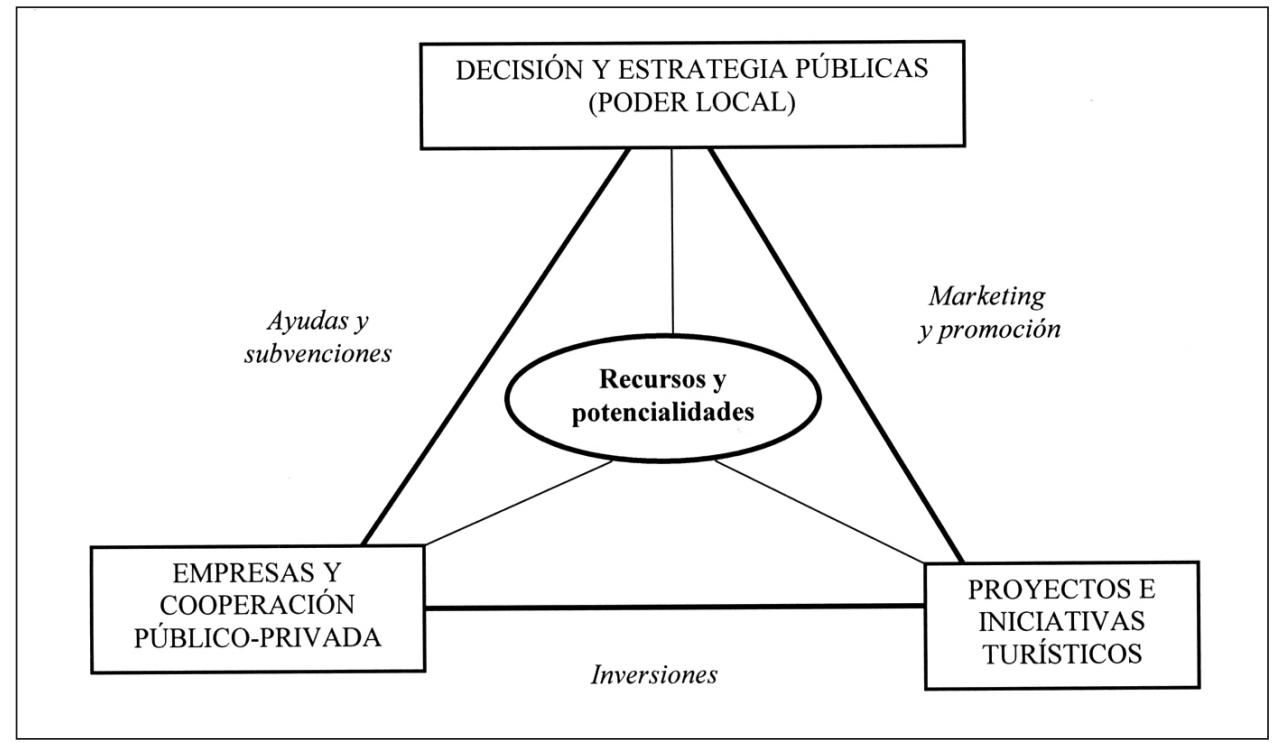

FIGURA 1: El entramado estratégico del desarrollo turístico local

Elaboración propia 
Los ámbitos regionales constituyen escenarios adecuados para captar el sentido de las tendencias variables que se aprecian en el territorio, máxime cuando los factores de diferenciación observados entre sus ciudades principales se traducen en especificidades internas que evidencian el alcance de los rasgos que respectivamente las definen y las complementariedades que al propio tiempo se establecen entre ellas. De ahí el interés de aprovechar el caudal de posibilidades interpretativas que ofrece la Comunidad Autónoma española de Castilla y León, la más extensa superficialmente de la Unión Europea, para valorar el significado del binomio turismo-ciudad en función de las peculiaridades que ofrecen, dentro del conjunto, las ciudades que ocupan un lugar secundario dentro de los destinos turísticos prevalentes y en las que no es difícil percibir tendencias que, desde la perspectiva que nos ocupa, tratan de conciliar la voluntad de afianzamiento de sus ventajas comparativas en materia turística con el aprovechamiento de las sinergias que a este respecto se derivan de las interacciones provocados por los flujos turísticos que tienen lugar en el seno del propio espacio regional.

\section{La dimensión turística de las ciudades capitalinas de Castilla y León: una aproxima- ción a la diversidad de posibilidades y tendencias en un escenario de gran calidad patrimonial}

La descentralización territorial del poder ha dado lugar en España a la creación de un escenario decisional en el que las Comunidades Autónomas desempeñan un papel fundamental en la gestión de las potencialidades turísticas, plenamente incorporadas a su ámbito de competencias, en virtud de lo establecido en el Art. 148.18 de la Constitución. Por tanto, resulta inexcusable aludir a las estrategias promovidas desde los gobiernos regionales para entender el impresionante avance que, tanto en términos de cantidad como cualitativamente, ha tenido lugar en la valorización de los recursos de que disponen los territorios concebidos a esta escala para acometer, ya sea en el marco de sus políticas globales de desarrollo regional o como factores de impulso y motivación de las administraciones locales, ambiciosas operaciones de acondicionamiento y proyección turística, que se manifiestan en numerosas opciones estratégicas.

La magnitud e importancia que el patrimonio cultural alcanza en Castilla y León (Blanco et al., 2003) convierte a esta región en un expresivo campo de experimentación de las políticas públicas orientadas al desarrollo turístico, sobre la base del amplio abanico de las posibilidades permitidas por el turismo interior. Una prueba fehaciente de la pluralidad de tendencias surgidas en este contexto territorial la ofrece el conjunto de las nueve ciudades que ocupan la primacía en la estructuración del sistema urbano regional precisamente por su condición de capitales provinciales. Considerarlas como referencias a tener en cuenta resulta pertinente por un doble motivo: de un lado, porque simbolizan los dinamismos y tendencias de cambio dominantes en sus respectivas provincias, donde ejercen una responsabilidad articuladora de primer orden, como centros polarizadores del crecimiento y dotadas al tiempo de la trama de servicios que posibilitan la atención de los flujos asociados a la demanda viajera; y, de otro, porque, debido a los factores y tendencias señalados, acusan en mayor medida que los núcleos situados por debajo de su nivel funcional los impactos inducidos por las actividades de ocio y recreación, de suerte que representan en el espacio las diferentes modalidades de transformación de que es susceptible una ciudad obligada a integrar en sus propias lógicas de crecimiento y adaptación urbanística las necesidades requeridas por una corriente caracterizada por su fuerte presión cualitativa.

Ahora bien, los contrastes de escala y la especificidad de sus rasgos patrimoniales respectivos explican la existencia de una tipología de situaciones que, como encuadre general 
de la cuestión que centra este trabajo, conviene precisar. De una parte, y como se recoge en el Cuadro 1, cabe resaltar las diferencias observadas respecto a la magnitud que en cada una de ellas alcanza las cifras de visitantes, que, aun referidas globalmente al conjunto de cada provincia, pueden ser orientativas del peso que a su vez corresponde a las capitales, en virtud del argumento señalado. La importancia ostentada por Salamanca resulta inequívoca, por más que tampoco se muestren alejados los datos ofrecidos por Burgos y León, que comparten con aquélla el rango de las provincias visitadas por más de un millón de personas. Claramente destacadas respecto a las demás, la secuencia ofrece en este caso un panorama gradualmente declinante, que revela, en principio, la desigual capacidad de motivación viajera que cada una de ellas suscita.

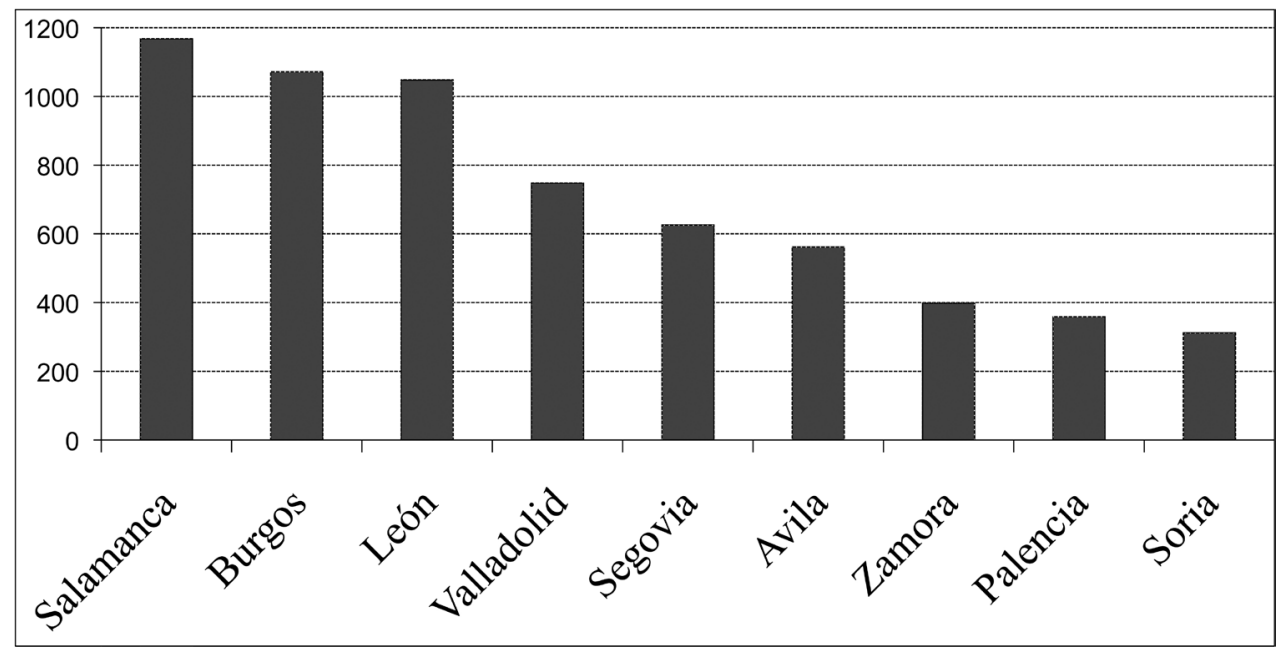

FIGURA 2. Viajeros registrados en Castilla y León (en miles)

Fuente: Boletín de Coyuntura Turística. Junta de Castilla y León. 2009

Sin embargo, no es posible hacer caso omiso, de otra parte, del criterio que justifica estos contrastes a partir de la relevancia que las distintas ciudades presentan desde el punto de vista de la estabilidad de los flujos viajeros que acceden a ellas y que han de ser entendidos de acuerdo con la magnitud de sus potencialidades de desarrollo turístico y que la propia transformación del espacio urbano, consecuente a las operaciones urbanísticas llevadas a cabo con este fin, se encarga de ratificar. En esencia, dichas potencialidades responden a la interacción producida entre tres factores fundamentales: la cantidad y la calidad de los recursos puestos a disposición de una demanda que valora su atractivo en función de la acreditación otorgada a gran escala; el prestigio alcanzado a partir de una buena proyección informativa, que en buena medida descansa en la solidez de su arraigo temporal; y la mejora de las condiciones de accesibilidad que facilitan el desarrollo de los movimientos turísticos en su beneficio. Las comprobaciones empíricas realizadas acerca de la posición que las distintas ciudades consideradas desempeñan en la ordenación del turismo urbano en Castilla y León permiten señalar cuatro situaciones jerárquicamente ordenadas desde el punto de vista de sus respectivas fortalezas turísticas, y que son presentadas de forma sucinta a fin de interpretar mejor el contexto donde se desenvuelven las ciudades en las que se centra este trabajo. 


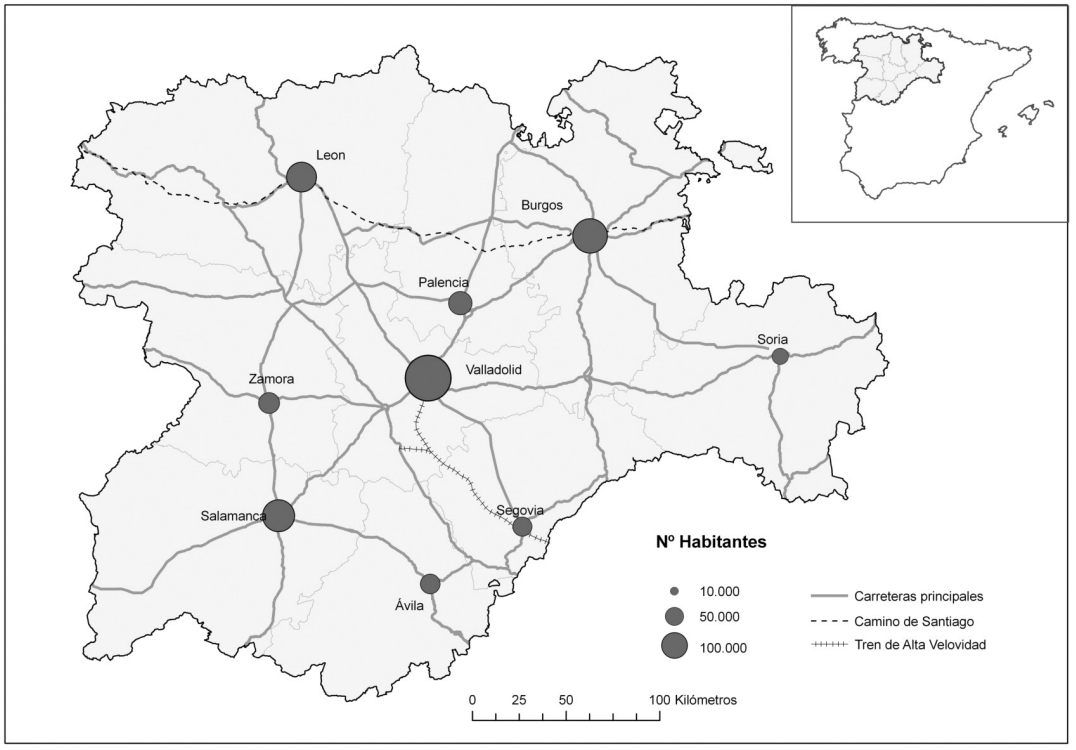

FIGURA 3. Red de capitales provinciales de Castilla y León y principales ejes de relación

- El lugar preeminente dentro de la serie corresponde sin duda a Salamanca (154.462), Segovia (55.784) y Ávila $(58.245)^{3}$, que han de ser incluidas en el mismo rango por su condición de Ciudades Patrimonio de la Humanidad. Es evidente que esta categoría las proporciona, de entrada, un reconocimiento por parte del mercado turístico plenamente asegurado y sin soluciones de continuidad en el tiempo (Troitiño y Troitiño, 2009). La primacía asignada a Salamanca, como la ciudad de mayor potencial turístico de la región y una de las principales de España, obedece a la extraordinaria calidad de su patrimonio arquitectónico, identificado con el legado del barroco, y a la estabilidad de una oferta de consumo cultural cimentada en torno a la capacidad de iniciativa desplegada hace mucho tiempo por su Universidad. Son los pilares en los que se apoya la parte sustancial de la economía salmantina y el tratamiento de que es objeto el centro histórico, cuya excepcionalidad estética resalta en una trama urbana que, al margen de él, adolece de numerosas deficiencias urbanísticas, hasta el extremo de crear dos espacios de relación y convivencia netamente diferenciados. La menor entidad demográfica y un planeamiento moderno mejor concebido en sus relaciones con la ciudad histórica han hecho posible la pervivencia de la imagen atractiva que presentan Segovia y Ávila, dotadas de elementos monumentales emblemáticos (acueducto romano y murallas medievales, respectivamente), además de edificios religiosos y civiles bien conservados y rehabilitados, que ejercen una función catalizadora de primer orden para el visitante, que accede a ellas, mediante desplazamientos de corta duración, aprovechando la cercanía de Madrid, como gran foco de demanda. La entrada en funcionamiento en diciembre de 2007 de la línea de alta velocidad ferroviaria que une la capital de España con Segovia ha acentuado aún más esta tendencia, paralela a la mejora de la comunicación por autovía que la une con Valladolid.

3 Con el fin de tener una idea de su dimensión demográfica, entre paréntesis se señala la población residente en las distintas ciudades, de acuerdo con las cifras de población referidas al 01/01/2010 y suministradas por el Instituto Nacional de Estadística. 
- La consideración de las ciudades de Burgos (178.574) y León (134.012) en un segundo nivel obedece a las sensibles analogías que es posible observar entre ellas. Si por su localización geográfica constituyen puntos esenciales de encrucijada en los extremos noroeste y nororiental de la región, lo que les proporciona una excelente renta de situación como ciudades de tránsito muy frecuente en las comunicaciones del norte de España, ambas ofrecen desde el punto de vista turístico elementos muy valorados. Entre ellos cabría destacar el atractivo de sus catedrales góticas y su pertenencia a la ruta del Camino de Santiago Francés, Patrimonio de la Humanidad desde 1993, y convertido en un vector turístico de primera magnitud a lo largo de todo su recorrido, con manifestaciones espaciales muy relevantes en el tramo que enlaza ambas ciudades. Con el tiempo, al acervo patrimonial heredado de la Edad Media y a la calidad urbanística de las transformaciones llevadas a cabo en el siglo XIX y comienzos del XX se suman operaciones de gran envergadura museística, como son las que han dado origen al Museo de Arte Contemporáneo (MUSAC) en León y al de la Evolución Humana en Burgos, asociado en este caso a los excepcionales hallazgos paleontológicos encontrados en la cercana Sierra de Atapuerca, y que han hecho del Museo burgalés - y del complejo configurado y organizado en torno a él- una de las referencias culturales y científicas más importantes del mundo en el campo de la arqueopaleoantología humana (Manero, 2011)

- Por su parte, la ciudad de Valladolid (315.522) merece una consideración específica. Se trata del espacio urbano de mayor entidad demográfica de la región, con una posición de centralidad, física y funcional, que no ha cesado de afianzarse tras su consolidación como capital de la Comunidad Autónoma a partir de 1987. Aunque dotada de elementos patrimoniales de gran significación en la historia de la arquitectura y del arte (Plaza de San Pablo, Plaza Mayor, Universidad, Museo Nacional de Escultura, Museo de Arte Oriental, Iglesia de la Antigua...) y escenario de eventos internacionalmente reconocidos (Semana Santa, Semana Internacional de Cine), su proyección turística no comienza a ser operativa hasta comienzos del siglo XXI, cuando se acomete, con resultados aún por evaluar, una estrategia decidida en esta dirección, con el fin de ampliar la imagen de una ciudad tradicionalmente ligada a su personalidad industrial y de escasa o esporádica relevancia turística. Los pasos dados en este sentido han sido tan firmes como acelerados, pues así se testimonian en las numerosas actuaciones encaminadas a la mejora cualitativa del centro histórico (peatonalización, rehabilitaciones integradas, recuperación de los espacios públicos, etc.) coincidentes en el tiempo con el proyecto de instalación de la línea ferroviaria de alta velocidad Madrid-Segovia-Valladolid, cuya puesta en servicio en diciembre de 2007 ha introducido un factor de estímulo importante, aunque aún no suficientemente cuantificado, en el reconocimiento de los potenciales turísticos vallisoletanos ${ }^{4}$. De ahí la importancia concedida a la elaboración y puesta en marcha del Plan Estratégico de Turismo (20102014), empeñado en perfilar una imagen identificada, entre sus valores esenciales, con el patrimonio histórico, con la gastronomía, con su relevancia como ciudad de congresos y con la figura del escritor Miguel Delibes, en el que se apoya una de las rutas urbanas - la «ruta del hereje»- más apetecidas.

4 Con todo, y según un informe de la Cámara de Comercio el número de viajeros entre Madrid y Valladolid en el primer año de funcionamiento del AVE se incrementó en un $30 \%$ respecto al anterior, testimonio igualmente avalado por la Asociación de Hosteleros que, pese a no haberlo cuantificado con precisión, asegura que desde la llegada del tren de alta velocidad «se ha notado en los fines de semana un aumento muy sensible de la cifra de negocio, particularmente en el campo de la restauración», aspecto que también se aprecia en los visitantes al Museo Nacional de Escultura y en los recorridos organizados por «la ruta del hereje», cuya cifra, según la Oficina de Turismo municipal, se ha casi triplicado en ese periodo. 
— Finalmente, la ciudades de Zamora (65.998), Palencia (82.169) y Soria (39.838), en las que se centra este artículo, forman parte de una categoría cuyos rasgos de coherencia vienen dados por dos tendencias que tienden a contrarrestarse. Pues si una de ellas tiene que ver con la posición ostensiblemente secundaria que las tres ocupan todavía en la magnitud global de los movimientos turísticos desplegados en Castilla y León, no es menos cierto que a la par tratan de fortalecer, no sin esfuerzo y con un empeño institucional muy fuerte, las bases de su personalidad cultural a fin de asumir, pese a su situación menos favorable de partida, los mismos desafíos que afectan al conjunto, con el que tienden a asimilarse al amparo de las favorables perspectivas existentes en el espacio regional.

\section{Entre el insuficiente conocimiento exterior de sus posibilidades y la voluntad de- cidida de acreditación turística: los casos de Zamora, Palencia y Soria}

Las consultas efectuadas en los tres casos (Oficinas de Turismo de los Ayuntamientos respectivos) ponen de manifiesto una estrategia basada en principios y directrices similares, cuya materialización aparece además acompasada en el tiempo. No se trata de emular miméticamente a las ciudades que ostentan las posiciones más destacadas en la serie regional sino de alumbrar líneas de actuación propias, incluso en algunos casos con pretensiones de originalidad, a sabiendas de que la diferenciación creativa puede suponer una garantía de proyección más plausible, sin perder al propio tiempo la perspectiva de las posibilidades que pudieran derivarse de su complementariedad respecto a la oferta y a los recursos presentados por ciudades próximas (Salamanca y Valladolid en el caso de Zamora; Valladolid y Burgos en el de Palencia), con las que poder mantener sinergias susceptibles de redundar en su beneficio ${ }^{5}$. En esencia, y a partir de las comprobaciones efectuadas, puede decirse que sus estrategias se decantan esencialmente en torno a dos líneas de actuación, ambas unidas por un denominador común: la voluntad política de poner en valor el patrimonio disponible, mediante el engarce pretendido entre los recursos tradicionales y otros de nuevo cuño, para de ese modo diversificar sus opciones de consumo cultural tratando de imbricar así tradición y modernidad.

\subsection{La progresiva adecuación del patrimonio construido de valor histórico a las pers- pectivas (crecientes) de la demanda turística}

Es evidente que el reclamo inducido por los recursos de valor histórico-artístico existentes en estas ciudades no es un fenómeno nuevo, pues su valor está ampliamente reconocido en el catálogo de bienes culturales de interés con que cuenta la región de Castilla y León. Sin embargo, su utilidad en el proceso de dinamización económica y social se ha visto condicionado por dos factores que no son tan manifiestos en el resto de las ciudades anteriormente consideradas.

De un lado, más que una visión global de lo que representaba la suma de elementos disponibles, se ha tenido de ellos hasta bien avanzados los años ochenta del siglo XX una perspectiva insuficiente, fragmentaria y disociada, que centraba la atención en aspectos singulares, al margen de una apreciación global y objetiva del conjunto, en sí mismo mi-

5 La situación periférica en que se encuentra la ciudad de Soria determina una dificultad estructural para beneficiarse, como sucede en las otras dos, de los efectos difusores de la demanda turística provenientes de las ciudades más potentes en este sentido y con las que mantienen estrechos vínculos de cercanía favorecidos por una conectividad muy alta. En el caso se Soria el problema se acentúa por la lentitud de las mejoras aplicadas a las infraestructuras por carretera que han de facilitar una mejor integración de dicha ciudad con Valladolid a través del valle del Duero. 
nusvalorado. Es lo que hasta hace bien poco ha sucedido en la ciudad de Zamora, cuya personalidad turística quedaba eminentemente circunscrita a las actividades organizadas en torno a la Semana Santa, el más poderoso catalizador del turismo zamorano, pese a su carácter episódico, y que, aparte de los actos propios del momento de celebración, se había dotado desde 1964 de uno de los primeros museos españoles sobre el tema, mucho antes de que se la concediera el rango de Fiesta de Interés Turístico Internacional. Su prevalencia eclipsaba y minimizaba a la vez las demás posibilidades latentes de la ciudad, que adolecían de falta de atención o de la consideración debida en el caso de las iglesias de estilo románico distribuidas en el centro urbano donde enriquecen sobremanera - en un elenco que es al tiempo único, por su número, en las ciudades europeas - la calidad patrimonial de la ciudad y, lo que era más grave, el abandono en que se encontraba el núcleo histórico, que establecía una solución de continuidad poco atractiva en el recorrido que enlaza la Plaza Mayor con la Catedral y el entorno del Castillo. En el mismo sentido, cabría aludir a la imagen ofrecida por Soria, muy condicionada, como se ha dicho, por su alejamiento físico respecto a los ejes de comunicación principales, y que sin duda contribuía a acentuar la sensación de lejanía con que se la contemplaba en el contexto de la región. Subestimada durante mucho tiempo la relación entre la ciudad y la presencia en ella (1907-1912) del poeta Antonio Machado, sus perspectivas como espacio canalizador de la atención turística quedaban restringidas a referentes puntuales, atendidos en visitas efímeras, como han sido los proporcionados por manifestaciones románicas de gran calidad (San Juan de Duero, Santo Domingo) o por el factor de singularidad que aportaba el conocimiento directo del Museo Numantino, resultado de la integración del Museo Provincial y del Museo Numantino, concebido éste desde 1914 para albergar los restos arqueológicos de la villa ibérica de Numancia, aunque su inauguración no tuviera lugar hasta 1919. Y por lo que respecta a la ciudad de Palencia, la nota de distinción ha estado siempre muy marcada por su Catedral gótica, popularmente denominada «la bella desconocida», sin que a la par acompañaran otros argumentos justificativos del recorrido por un casco urbano identificado con su Calle Mayor, que apenas motivaba, a quien lo hiciera, sino una visita fugaz, y, menos aún, por el amplio elenco de edificios religiosos (Monasterio de Santa Clara, Convento de San Bernardo, San Juan Bautista...) sumidos en el desconocimiento para la mayoría de los visitantes que accedía a la ciudad bañada por el Carrión o se interesaban por ella.

De otro lado, destaca, en los casos que nos ocupan, un segundo factor no menos llamativo. Se relaciona con la constatación de que las tendencias turísticas se han visto en algunas de estas ciudades más neutralizadas que complementadas por la importancia de los atractivos que encierran sus respectivos ámbitos provinciales, aunque esta tendencia no sea percibida en Zamora con la misma intensidad que en las otras. En cambio, sí cabría decir que durante mucho tiempo las ciudades de Palencia y Soria han tenido una importancia secundaria respecto a los flujos foráneos motivados en las provincias por elementos culturales de gran valor tanto en emblemáticas comarcas como en municipios específicos del mundo rural. Esta situación resulta ostensible en el primer caso, donde el reconocimiento de la provincia como ámbito de interés y motivación ha prevalecido sensiblemente sobre el que pudiera merecer la capital, mucho más modesto. La calidad y variedad de las manifestaciones del románico, dispersas en el tramo palentino del camino de Santiago (Frómista, Carrión) o en sus proximidades (Amusco) como en la montaña septentrional - donde sobresale el conocido como «Románico Norte» (Aguilar de Campoo, Cervera de Pisuerga) - han sido tradicionalmente puntos de atención privilegiada, capaces de atraer una corriente turística significativa, que al tiempo se ha decantado también por otros elementos que enriquecen la dotación patrimonial de los espacios rurales como el Canal de Castilla o el espectacular vestigio arqueológico formado por la villa romana de La Olmeda (Saldaña). Y en el mismo 
sentido, son muchos los testimonios que en el territorio soriano se han superpuesto con holgura a los ofrecidos por la capital de la provincia. Bastaría hacer mención al formidable elenco de posibilidades existentes en sus comarcas sudorientales (Burgo de Osma, Berlanga, Almazán, Tiermes...) para percatarse del marcado sesgo que quienes visitan el ámbito soriano muestran por este sector del mundo rural (Bachiller, 1996, 282).

Ahora bien, tales condicionamientos, que han relegado durante mucho tiempo a un segundo plano la proyección de los centros capitalinos como focos de atención turística, han sido progresivamente superados a medida que los agentes responsables - tanto institucionales como privados - asumieron la necesidad de incorporarlas a la fase expansiva del turismo regional, aprovechando, como es obvio, el efecto inductor provocado por las ciudades con mayor entidad en este sentido. No cabe duda que el proceso no puede entenderse, en principio, al margen del apogeo alcanzado por las políticas públicas de apoyo al turismo interior en España (Manero, 1997, 319), que experimenta en la última década del siglo XX un despegue significativo hasta ofrecer en la siguiente una tendencia fuertemente alcista, que ofrece en Castilla y León algunas de sus magnitudes más expansivas, referidas tanto al turismo rural como al urbano (Blanco et al., 2003). Asimismo en esta tendencia desempeñan también un papel decisivo las iniciativas acometidas por el poder local y provincial, claramente manifiestas en las campañas ciudadanas a favor de la defensa, protección y puesta en valor de sus recursos patrimoniales, y los programas amparados en la intervención pública descentralizada, merced a las competencias exclusivas asumidas por la región, que concibe el turismo como uno de los pilares en el que basar sus estrategias de desarrollo, y que al tiempo sintonizan con la intencionalidad de los programas de fomento del turismo (Planes de Excelencia y Planes de Dinamización Turística, que a partir de 2005, y definidos como «herramientas de cofinanciación de las estrategias turísticas», se engloban bajo la denominación de «Planes de Dinamización del Producto Turístico») impulsados desde el gobierno central y contando asimismo con un significativo apoyo de los fondos estructurales aportados por la Unión Europea.

En cualquier caso, la confluencia de estos tres factores - es decir, efectos de emulación y sinergia entre ciudades, empeño ciudadano, y fuerte respaldo político y financiero institucional, en el que se implican los cuatro niveles de la Administración pública (Estado, Región, Diputación y Municipio $)^{6}$ - justifica el importante salto cualitativo emprendido en la primera década del siglo XXI por estas ciudades de menor relevancia turística, que hacen suyas las posibilidades ofrecidas para abordar ambiciosas actuaciones en torno a dos ejes principales: de un lado, la recuperación de elementos subvalorados del patrimonio construido, sustentados en las potencialidades preexistentes, y que ahora se redescubren y proyectan con decidida voluntad de política urbanística, y, de otro, la creación de nuevos productos de reclamo turístico, en sintonía con la voluntad de impulsar manifestaciones culturales apoyadas en proyectos susceptibles de atraer la atención del mercado, y que también acaban dejando su impronta en la transformación del espacio urbano.

El ejemplo de Zamora resulta ostensible tras la puesta en marcha en 2001 de las operaciones asociadas al Plan Especial del Centro Histórico, posteriormente reelaborado en 2010,

6 Particular atención debe reconocerse a la estrecha cooperación llevada a cabo en este proceso por los Ayuntamientos capitalinos y la Diputaciones Provinciales, comprometidos en la adopción de medidas y programas coordinados que con frecuencia presentan conjuntamente y que dan fiel idea de hasta qué punto se trata de imbricar las perspectivas turísticas de la ciudad con las que presenta el ámbito provincial en el que se integra. Esta relación fraguada entre ciudad y provincia, y claramente explícita por parte de sus principales responsables, se ha convertido así en la estrategia más apetecida por ambas partes, tal y como he podido comprobar en numerosas ocasiones y particularmente en los actos que, con tal fin, se organizan para dar a conocer las posibilidades turísticas respectivas con motivo de la importante Feria de Turismo Interior (INTUR) realizada anualmente en Valladolid en el último fin de semana de noviembre. 
una de cuyas aportaciones más relevantes consiste en facilitar una mejor integración del conjunto urbano con las riberas del Duero. Su ejecución, a lo largo de diferentes fases, ha supuesto la recuperación de un entorno con evidentes síntomas de deterioro y con escasa valoración como espacio residencial. La coincidencia en el tiempo del Plan urbanístico con el de Excelencia Turística (2002) ha permitido establecer entre ambos un engarce técnico y funcional, capaz de potenciar, social y culturalmente, el entorno de la Catedral mediante operaciones destinadas a promover la atención del visitante y a acreditar para el propio ciudadano el valor de ese espacio como área de esparcimiento y de vivienda ${ }^{7}$. De ahí que simultáneamente hayan sido acometidas las intervenciones de rehabilitación de edificios con fines residenciales y las que se han traducido en el acondicionamiento de numerosos bienes arquitectónicos, como es el caso de las iglesias románicas, embellecidas y debidamente integradas en las rutas urbanas o la recuperación de las ruinas del Convento de San Francisco para instalar, en el marco que las aprovecha, la sede de la Fundación Rei Alfonso Henriques, dedicada al fortalecimiento de los vínculos culturales y científicos con Portugal.

Al propio tiempo, el centro histórico afianza y mejora sensiblemente su condición de espacio público con el reacondicionamiento del entorno del Castillo, que aporta así una dotación adicional a la visita a la Catedral (Foto 1), o la construcción de edificios de gran significado cultural, como es el Centro de Interpretación de las Ciudades Medievales y el Museo Etnográfico (2002), integrado en la red de Museos de la Junta de Castilla y León ${ }^{8}$. No menor importancia reviste en este caso la utilización del legado modernista al acervo de la arquitectura urbana más valorada, con la que se trata de presentar las manifestaciones artísticas de un período significativo de la historia de la ciudad que establece un contrapunto muy interesante respecto a las de origen medieval. Con el enfático nombre de la «Ruta europea del Modernismo», e identificándose como «la ciudad con la mayor concentración de arte románico de Europa», pone a disposición del visitante un elenco valioso de construcciones que, conservando su función habitacional, evocan las múltiples particularidades de la arquitectura decimonónica.

Dentro de directrices similares se inscriben las mejoras introducidas en Palencia y Soria. En ambas, y como ha sucedido de manera generalizada, la aplicación del planeamiento urbanístico orientado al conocimiento, protección y gestión integrada del centro histórico ha evolucionado en sintonía con la voluntad de impulsar una imagen más atractiva, en la que confluyeran los elementos religiosos de la herencia medieval - renovados en su presentación estética e informativa y enaltecidos en el entorno en el que se encuentran - y aquellos que, formando también parte de la historia urbana, no habían recibido el reconocimiento merecido. La instalación del Centro de Interpretación del Románico (2008) en la Huerta de Guadián de Palencia es una iniciativa paralela con la creciente atención prestada a la figura de Jerónimo Arroyo, artífice de algunos de las representaciones más valiosas de la arquitectura modernista palentina (Diputación Provincial, Instituto Jorge Manrique, Colegio Villandrando, Casa de Flora Germán y Fulgencio García...), y que ha acabado configurando - junto a los edificios diseñados por Jacobo Romero, otro de los grandes arquitectos palentinos - una ruta urbana específica, de gran valor cultural, que contribuye a personalizar y dar realce al catálogo turístico de la ciudad. Y, de forma más puntual, cabría

7 La potenciación de este ámbito se concibe como una de las prioridades básicas de la Revisión del Plan, teniendo en cuenta que la más importante perspectiva de desarrollo que se ofrece a la ciudad es la expansión en el ámbito turístico en base a los atractivos culturales e histórico-artísticos existentes. Documento de Revisión del Plan General de Ordenación Urbana de Zamora, 2001, p. 90.

8 Comparte este rango con el Museo de la Evolución Humana, ubicado en Burgos, con el de Arte Contemporáneo (MUSAC), en León, y con el de la Siderurgia y la Minería, instalado en el municipio leonés de Sabero. 


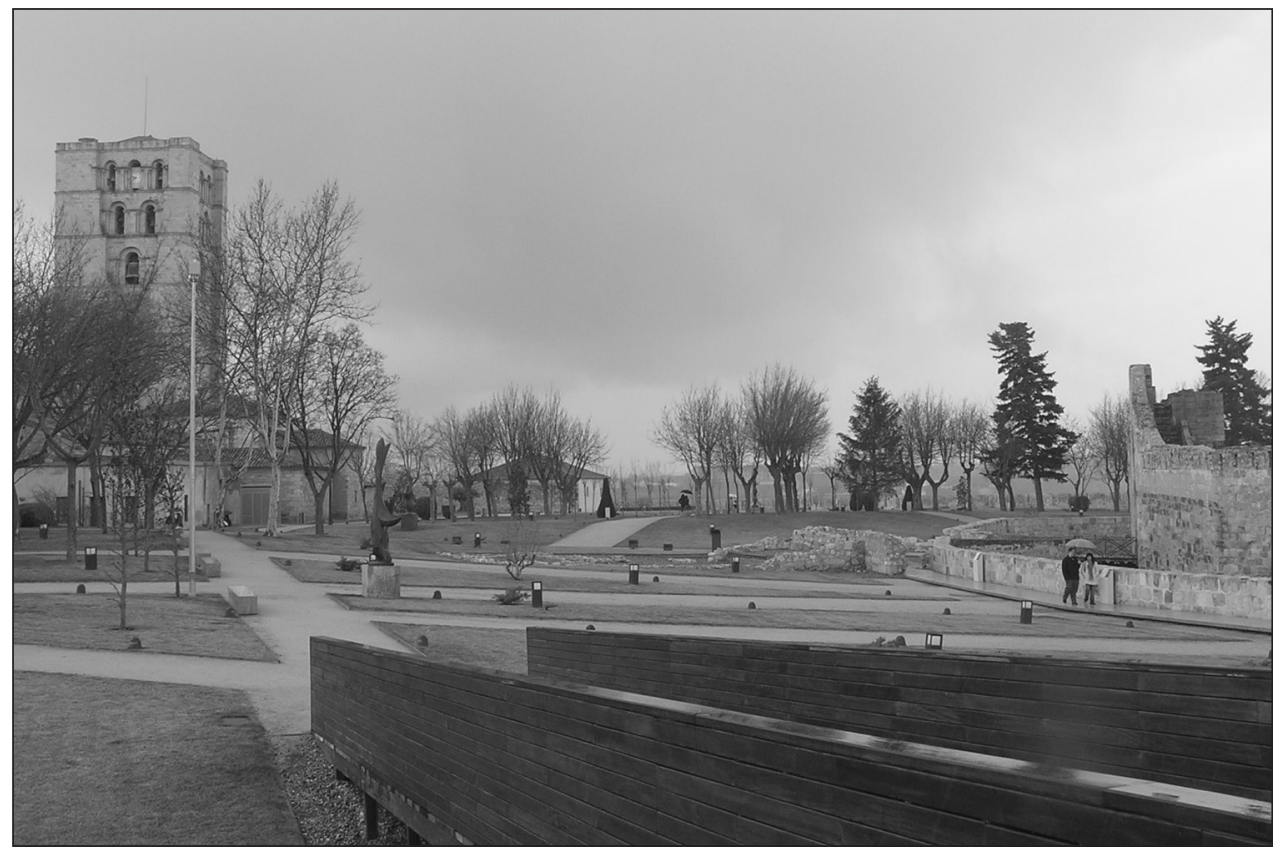

Fото 1. Zamora. La configuración de un nuevo espacio público en la ciudad histórica, organizado en función de los elementos arquitectónicos más emblemáticos.

aludir, en lo que concierne a Soria, el significado concedido a la ampliación del Museo Numantino, que se aborda a finales de los años ochenta del siglo XX, con la que se trata de culminar un ambicioso proyecto de renovación, que amplió sensiblemente la superficie con el fin de ofrecer la imagen de un lugar completamente renovado y representativo de la importancia del patrimonio arqueológico en el que se fundamenta.

\subsection{Diversificación e innovación del producto turístico. Hacia una recualificación integral del espacio urbano}

Si las bases sobre las que se asienta la personalidad turística de las ciudades consideradas aparecen sólidamente definidas por las medidas aplicadas a la ordenación y valorización de su patrimonio monumental de raíz histórica, no son desestimables tampoco los esfuerzos realizados para diversificar su oferta mediante la incorporación de nuevos productos que, sumados a los anteriores, acaben configurando una dotación consistente que amplíe el conocimiento que de ellas se tiene y contribuya al propio a tiempo a facilitar una inserción más consistente en la estructura del turismo urbano regional. En esencia, la elaboración y puesta en marcha de nuevos productos con este fin no hace sino reconocer el campo de posibilidades permitido por los recursos naturales y culturales intrínsecos a las ciudades, suficientemente conocidos con anterioridad pero nunca asumidos como ventajas comparativas, en ocasiones incluso singulares, dignas de ser valorizadas. Pero, más allá del esfuerzo de imaginación que ello implica desde el ámbito local donde se plantean, no cabe duda que estas iniciativas se apoyan tanto en un análisis previo de las estimaciones 
sobre su potencial interés en el mercado como en la aceptación de ideas provenientes de la experiencia comparada, que se nutre de los resultados obtenidos en iniciativas similares ya sea en el contexto español o europeo. Desde esta perspectiva, y sin agotar la pluralidad de opciones que pudieran presentarse, tres son los ejes principales hacia los que se orienta esta estrategia de apertura a nuevos horizontes en el diseño de la oferta.

Son interesantes, en primer lugar, las intervenciones efectuadas para dar a conocer la belleza y calidad de los paisajes construidos en torno al agua, elemento natural al que se asigna un valor patrimonial de primer orden. A este respecto, la confianza depositada en el río Duero unifica la atención otorgada en las dos ciudades que se identifican con su trazado. De modo preeminente, Soria trata de lograr mediante el acondicionamiento de las márgenes del río uno de los principales reclamos que justifiquen la visita, aprovechando la gran calidad paisajística del entorno que rodea la ciudad, como ambiciosas son también las pretensiones de Zamora de hacer de este curso de agua el símbolo de su personalidad ambiental, unida a la recuperación de las aceñas medievales. Y, como era previsible, la misma lógica inspira la voluntad del Ayuntamiento palentino de hacer suyas las ventajas de su proximidad al Canal de Castilla (Foto 2), una espectacular obra de ingeniería hidráulica iniciada en el siglo XVIII, que es incorporada a la oferta turística de la ciudad aprovechando los ramales que llegan hasta ella y como fundamento para la instalación de un «Museo del Agua» mediante la rehabilitación de naves destinadas al almacenamiento del cereal.

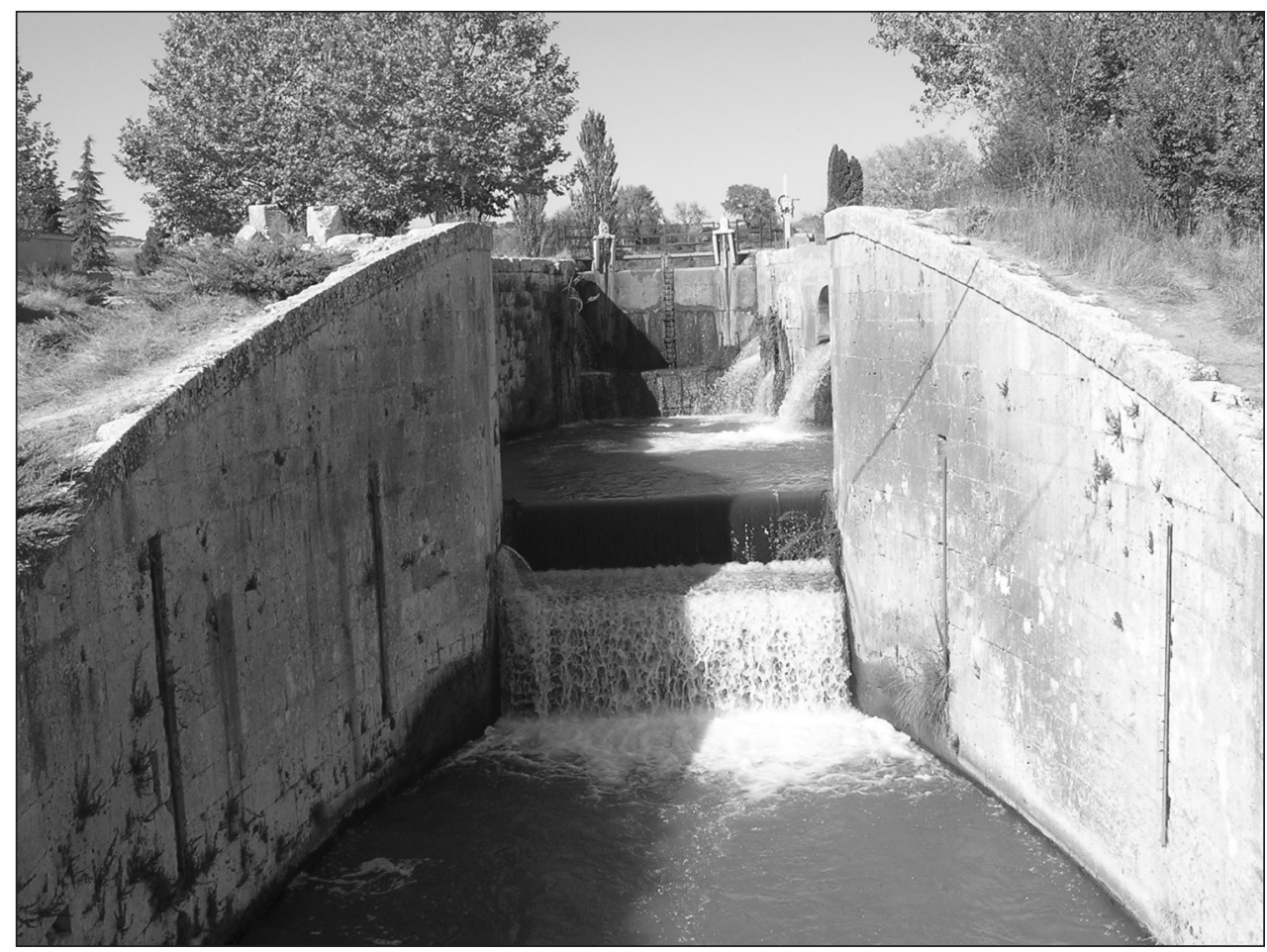

Fото 2. Palencia. El Canal de Castilla como elemento singular de atracción turística 
Por otro lado, las diferentes manifestaciones del Arte y Literatura favorecen la búsqueda de formas de plasmación en el espacio amparados en nombres emblemáticos de la creatividad cultural, y cuya obra trasciende con creces el ámbito de las ciudades que los reconoce y asimila como propios. Con este fin, Soria ha hecho suya, como uno de sus más preciados valores patrimoniales, la figura de Antonio Machado (Foto 3), ofreciendo los lugares donde residió y trabajó durante su estancia en la ciudad como testimonios de una época y de la relevancia cultural de uno de los más afamados poetas españoles, lo que ha llevado a considerar su imagen como el argumento central del Museo de los Poetas, proyectado en las instalaciones del edificio modernista donde se ubica el Casino. Al tiempo, la adaptación de una parte del edificio del Banco de España para la instalación del Museo Nacional de Fotografía del Paisaje, añade otro importante señuelo de interés cultural en el corazón mismo de la ciudad. Por su parte, las distintas facetas de la expresión artística permiten a Palencia albergar las interesantísimas piezas que evocan, en sendos Museos, el talento y la creatividad de Victorio Macho (escultura), Juan Manuel Diaz Caneja (pintura) y Jerónimo Arroyo (arquitectura), a semejanza de lo realizado por Zamora con la construcción del nuevo edificio que, al lado de la catedral románica, acoge parte de la espectacular obra del escultor Baltasar Lobo, realizada durante su exilio en Paris, donde falleció en 1993.

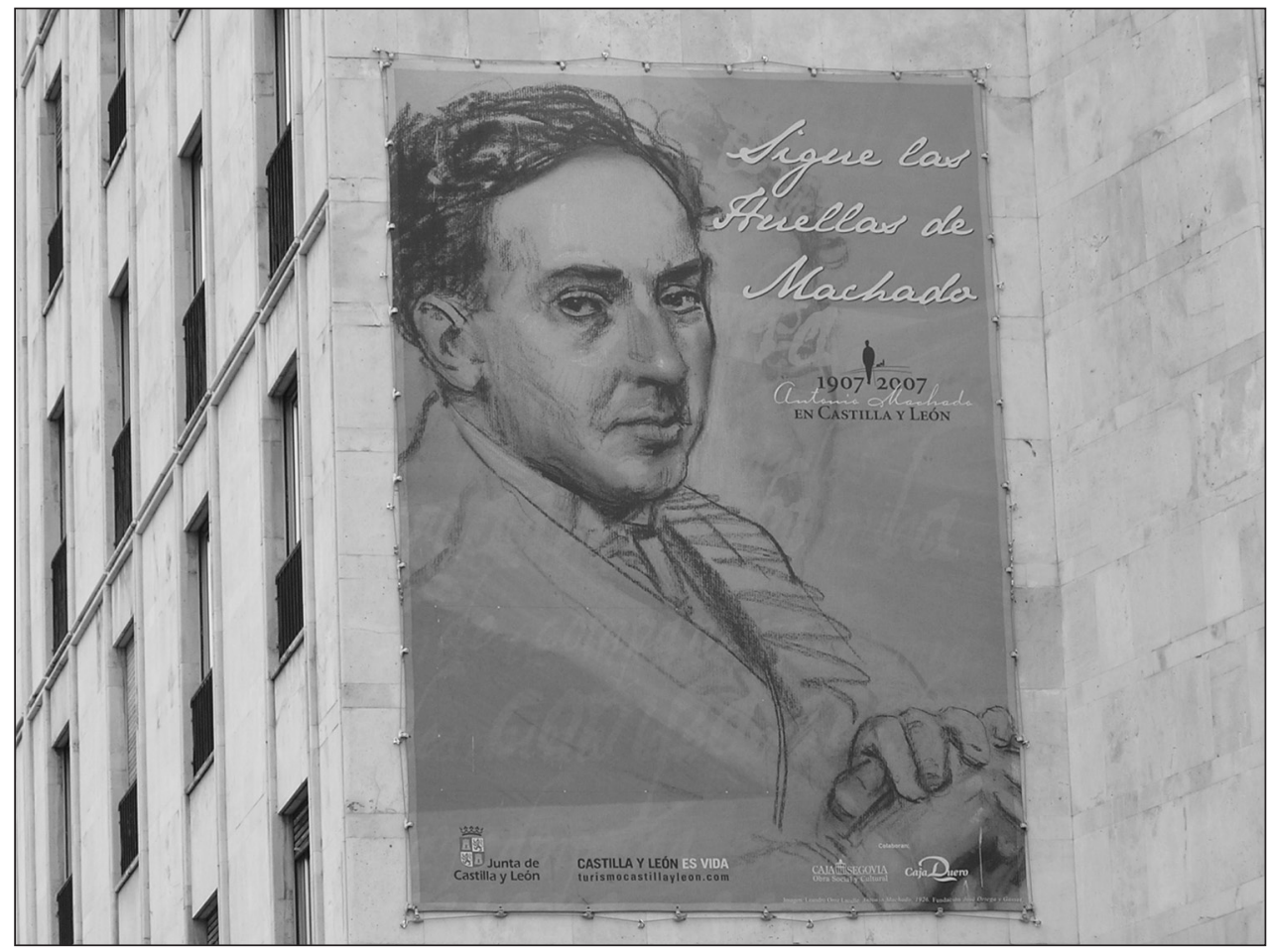

Fото 3. Soria. Una imagen de calidad en buena parte construida en torno a la figura de Antonio Machado

Y, finalmente, el abanico no cesa de ofrecer nuevos productos ante el señuelo que eventualmente pueden proporcionar eventos culturales, presentados como referencias a tener en cuenta, una vez conseguidos los niveles de aceptación que aseguran, o pueden asegurar, su 
continuidad en el tiempo. La música aparece así como la manifestación de una iniciativa capaz de movilizar los compromisos del poder local con tanto esfuerzo económico, apoyado en el patrocinio privado, como dedicación promocional. El «Otoño Musical Soriano», creado en 1993 y asociado a la figura del director Odón Alonso, acredita una experiencia que sólo cabe entender en función de la pluralidad de formas de expresión musical con que está concebido y con el alto nivel de calidad alcanzado, rasgo del que también participa El Festival Internacional de Música «Pórtico de Zamora», iniciado en 2004 y centrado en la música clásica. Y tampoco se puede omitir la positiva respuesta conseguida por el Festival «Enclave de Agua» que desde 2008 celebra en Soria los conciertos de Música Afroamericana, a los que se accede gratuitamente y que constituyen una aportación cultural impresionante en el entorno de la ciudad bañada por el Duero.

\section{Conclusiones}

No es posible entender el significado y alcance económico-espacial de las transformaciones urbanas contemporáneas sin aludir al impacto provocado por el turismo y los efectos inducidos que de él derivan. Se trata de un fenómeno que de manera generalizada orienta y galvaniza uno de los objetivos primordiales de las políticas públicas, del que, como es lógico, participan también las ciudades de reducida dimensión que tradicionalmente han ocupado una posición secundaria entre los destinos más apetecidos y valorados por la demanda. De hecho el proceso de diversificación ha proporcionado al turismo urbano, ejemplificado en las analizadas en este artículo, una nueva dimensión, más plural, abierta, creativa e innovadora, producto de la estrategia encaminada a fortalecer, aun consciente de los riesgos que entraña, sus posibilidades de atracción turística en un contexto de elevada demanda potencial como es el que ofrece la dotación patrimonial existente en Castilla y León.

A falta de hacer un estudio pormenorizado, y con la suficiente perspectiva temporal, de las tendencias propiciadas por este aumento de la oferta, las estimaciones cualitativas obtenidas in situ revelan que siguen siendo destinos claramente secundarios en el cómputo del turismo regional, con fuertes altibajos en la evolución de las visitas, cuyo incremento aparece supeditado a la celebración de eventos puntuales, que aseguran un momento alcista eminentemente coyuntural. De todos modos, esta propensión al carácter episódico de la demanda no impide, desde la perspectiva de los Ayuntamientos que ostentan la responsabilidad del proceso, abrigar expectativas prometedoras a medida que los productos puestos a disposición del mercado, y debidamente promocionados y gestionados, consigan ampliar el conocimiento de que son objeto aun a sabiendas de que los umbrales de rentabilidad alcanzados distarán mucho de corresponderse con la entidad de las inversiones realizadas. En cualquier caso, se asume que se trata de un proyecto de valorización turística a medio y largo plazo, cuya gestión va a requerir superar inercias, corregir insuficiencias en la promoción y lograr un engarce vigoroso en el entramado turístico de la región.

Sin embargo, la perspectiva pluridimensional con que se plantea justifica un interés que va más allá de las actuaciones puntuales o de los programas específicamente orientados a su dinamización. De hecho, si todas las medidas adoptadas persiguen atraer la demanda, bien de manera directa o indirectamente (aprovechando los desplazamientos turísticos realizados en el conjunto del espacio regional), no es menos cierto que al propio tiempo las políticas públicas han concedido una importancia primordial a la renovación y embellecimiento del espacio mediante la aplicación de los instrumentos permitidos por la planificación urbanística, cuyo impacto se acusa simultáneamente en la modificación del tejido urbano y en las operaciones de rehabilitación arquitectónica, determinantes para que la ciudad recupere la imagen de calidad y distinción que durante mucho tiempo 
había quedado relegada a un segundo plano en las prioridades del municipio. Lo que es evidente es que estas estrategias - por más que a veces adolezcan de una visión esteticista y no logren eclipsar los elementos visuales de actuaciones heredadas, menos sensibles con la calidad que ahora se pretende - han contribuido a transformar el espacio urbano, amparado en las iniciativas relacionadas con la adaptación y mejora de los elementos más valiosos del patrimonio edificado, en clara sintonía con los enfoques actualmente aplicados en las ciudades a la mejora de la calidad del uso turístico de los espacios públicos (ODIT, 2008, 34).

La relación entre turismo y ciudad ha traído consigo una doble incidencia: de un lado, de carácter cuantitativo, por cuanto ha dado origen a la ejecución de operaciones inmobiliarias que han requerido un considerable esfuerzo financiero; y, de otro, se refleja desde el punto de vista cualitativo, en la medida en que han introducido en las ciudades nuevas formas de expresión arquitectónica, responsables de una modificación sensible de las percepciones que tradicionalmente tenían de la ciudad tanto los propios residentes como quienes las visitaban. En realidad, en ningún caso estas construcciones, que amplían el inventario arquitectónico y museístico, se han traducido en actuaciones de gran impacto. Ha primado la pequeña escala mediante intervenciones que al tiempo han hecho posible la recuperación del espacio público, el descubrimiento de bienes patrimoniales abandonados o poco estimados para favorecer, en suma, una mejora en la imagen valorativa de la ciudad tradicional, donde los impactos no han conseguido distorsionar la armonía del conjunto. Todo ello a la espera de que los objetivos pretendidos con el relanzamiento de su prestigio como destino turístico se consolide más como opción apetecida que meramente complementaria de la de otras ciudades próximas, más vigorosas en este sentido.

\section{Bibliografía}

ALONSO, M. R. (1997): «La identificación de los espacios culturales en el ordenamiento español y la necesidad de integrar su tratamiento en el marco de la ordenación territorial», en Patrimonio Cultural y Derecho, ${ }^{\circ}$ 1, pp. 101-119.

BACHILLER, J. (1996): Espacios rurales desfavorecidos. Las transformaciones del suroeste soriano. Secretariado de Publicaciones de la Universidad de Valladolid. Valladolid, $356 \mathrm{pp}$.

BLANCO, A., FRANCO, F., HERNÁNDEZ, A., HERRERO, L. C., HORTELANO, L.A. y MANERO, F. (2003): Castilla y León. Presente y futuro del turismo. Estudios de la Fundación Encuentro y Caja España. Madrid, 324 pp.

CALLE, M. (2006): La ciudad histórica como destino turístico, Ariel. Barcelona, 304 pp.

CALLE, M. (2008): «El turismo en las políticas urbanas. Aproximación a la situación de las ciudades españolas». En IVARS, J.A.; VERA, J.F. (coord.). Espacios Turísticos. Mercantilización, Paisaje e Identidad. Actas del XI Coloquio de Geografía del Turismo, Ocio y Recreación de la AGE. Universidad de Alicante, pp. 507-529

EUROPEAN ASSOCIATION OF HISTORIC TOWNS AND REGIONS (2009): Guidelines. Sustainable Cultural Tourism in Historic Towns and Cities, Council of Europe. http:// urbact.eu/fileadmin/corporate/doc/EAHTR_guide.pdf

FEDERACIÓN ESPAÑOLA DE MUNICIPIOS Y PROVINCIAS (2009): Guía para la evaluación de las políticas culturales locales. FEMP. Madrid. http://www.femp.es/ files/120-18-CampoFichero/Evaluacionpol\%C3\%ADticas.pdf

GARCÍA, M.I., FERNÁNDEZ, Y. y ZOFIO, J.L. (2003): «The Economic Dimension of the Culture and Leisure Industry in Spain: National, Sectoral and Regional Analysis», en Journal of Cultural Economics, Vol. 27, 1. pp. 9-30. 
GARCÍA MARTÍNEZ, C. (2011): «La imagen en el turismo urbano: revisitando el Toledo romántico», en Cuadernos de Turismo, nº 27, pp. 437-453.

GREFFE, X. (1990): La valeur économique du patrimoine. La demande et l'offre de monuments. Anthropos. Paris. 253 pp.

LAW, C.M. (2002). Urban tourism: the visitor economy and the growth of large cities. C.M. LAW. London, 217 pp.

MANERO, F. (1997): «Experiencias de turismo interior: logros y fracasos desde la perspectiva del desarrollo local», en VALENZUELA RUBIO, M. (coord.): Los turismos de interior. El retorno a la tradición viajera. Madrid, Ed. Universidad Autónoma de Madrid (Col. Estudios, no 52). Madrid, pp. 307-329.

MANERO, F. (2000): «El significado económico-espacial de la rehabilitación urbana», en MiLlaruelo, J. y ORDUÑA, E.: Patrimonio artístico. Protección de conjuntos y edificios históricos. Ciudad Argentina. Buenos Aires, pp. 95-116.

MANERO, F. (2003): «El turismo como factor de cualificación espacial, identificación cultural y articulación del territorio castellano-leonés», en BLANCO, A. (Coord.): Castilla y León, presente y futuro del turismo, Estudios de la Fundación Encuentro y Caja España. Madrid, pp.135-168.

MANERO, F. (2011): «Patrimonio cultural y transformación urbana: ordenación espacial y proyección turística de los yacimientos arqueopaleontológicos de Atapuerca (Burgos)», en Cuadernos de Turismo, $\mathrm{n}^{\circ}$ 27, pp. 617-640.

MARCHENA, M. (1998): «Patrimonio y ciudad: nuevos escenarios de promoción y gestión del turismo urbano europeo», en MARCHENA GÓMEZ, M. (ed.), Turismo urbano y patrimonio cultural. Una perspectiva europea. Diputación de Sevilla. Sevilla, pp. 9-35.

ODIT FRANCE (2008): Améliorer la qualité d'usage touristique des espaces publics en ville. Des pistes de reflexión et de sensibilisation pour l'action. ODIT France. Paris, 92 pp.

PATIN, V. (1997): Tourisme et patrimoine en France et en Europe. La Documentation Française. Paris, 174 pp.

REYSSET, P. (2008): Aménager la ville. L'art d'habiter. Sang de la Terre. Paris, 190 pp.

SÁNCHEZ, E. (1999): «La protección del Patrimonio Cultural en el Derecho Español. Las competencias municipales», en Vivir las Ciudades Históricas: Urbanismo y Patrimonio Histórico, Fundación Cultural Santa Ana. Ávila, pp. 35-70.

SELBY, M. (2004): Understanding urban tourism: image, culture and experience. I.B. Tauris \& Co. Ltd., London, 228 pp.

TROITIÑO, M.A. y TROTIÑO, L. (2009): «Turismo y patrimonio en Castilla y León: las ciudades Patrimonio de la Humanidad (Ávila, Salamanca y Segovia) como destinos turísticos de referencia», en Polígonos. Revista de Geografía, no 19, pp. 145-178.

VIRASSAMY, C. (2002): Les pôles d'economie du patrimoine. La Documentation Française. Paris, $89 \mathrm{pp}$. 